CULTURA, LENGUAJE Y REPRESENTACIÓN / CULTURE, LANGUAGE AND REPRESENTATION · ISSN 1697-7750 • VOL. XV \2016, PP. 99-118 REVISTA DE ESTUDIOS CULTURALES DE LA UNIVERSITAT JAUME I / CULTURAL STUDIES JOURNAL OF UNIVERSITAT JAUME I

DOI: HTTP://DX.DOI.ORG/10.6035/CLR.2016.15.7

\title{
Los medios comunitarios, libres y ciudadanos en el Estado español. Territorios, tecnologías y valores ${ }^{*}$
}

Community, free and citizen media in Spain: territories, technologies and values

SERGIO VILLANUEVA BASELGA

UNIVERSITAT DE BARCELONA

ALEJANDRO BARRANQUERO CARRETERO

UNIVERSIDAD CARLOS III DE MADRID

JUAN RAMOS MARTÍN

UNIVERSIDAD DE SALAMANCA

Recibido: 11-10-2014

Aceptado: 07-03-2015

Resumen: Presente desde finales de la década de los años 70, el sector de los medios comunitarios, libres y ciudadanos ha tenido una enorme importancia en la construcción de espacios públicos alternativos, diversos y plurales y en la reivindicación de lenguas, culturas y resistencias activas minorizadas bajo diferentes formas de dominación. Sin embargo, a pesar de su importancia social, no existen análisis integrales acerca de la situación actual del sector. Este trabajo pretende ofrecer un panorama de análisis acerca de los actores presentes entre dichos medios, las formas de organización e interacción, la construcción de identidades alternativas y propias, el uso de las nuevas tecnologías y la penetración de los procesos de digitalización en sus estructuras y la construcción de valores comunes y formas propias de participación.

Palabras clave: medios comunitarios, medios ciudadanos, comunicación alternativa, tercer sector de la comunicación, comunicación para el cambio social, educación.

* Agradecemos, en primer lugar, el sustento económico que garantiza la sostenibilidad del proyecto por parte del Centro Reina Sofía sobre Adolescencia y Juventud. En segundo lugar, extendemos el agradecimiento al resto del equipo que ha permitido la consecución del proyecto «Los jóvenes y el tercer sector de la comunicación en España». Por último, agradecer el apoyo desinteresado a los organizadores y asistentes de los grupos focales realizados en Sevilla, Vitoria, Madrid y Barcelona que, aunque no son objeto de esta investigación, forman parte del proyecto. 
ABSTRACT: Since the late 70s, the sector of the community, free and citizen media has developed a huge importance in the improvement of alternative, diverse and plural public spaces and the claim of languages, cultures and resistances minorised by several forms of domination in the state. Despite its social importance, there is no analysis about the current situation of the sector. This work aims to provide an overview about the actors, forms of organization and interaction, the construction of alternatives and own identities, the use of new technologies, the penetration of the digitization process in its structures and the construction of common values and own forms of participation.

Keywords: community media, citizen media, alternative communication, third communication sector, communication for social change, education.

\section{Contextualización de los medios comunitarios, libres y ciudadanos en el Estado español}

Los medios comunitarios son medios a cargo de comunidades y colectivos ciudadanos con clara vocación de servicio a la comunidad y abiertos a la participación de la ciudadanía tanto en la elaboración de contenidos como en los procesos de administración y toma de decisiones. Estos medios forman parte de lo que también se conoce como el «tercer sector de la comunicación», puesto que, aunque su régimen de propiedad es privado, su gestión está a cargo de movimientos sociales y organizaciones sin ánimo de lucro. Sus objetivos son también distintos de los de los medios convencionales -el empoderamiento político, el refuerzo de identidades y de derechos humanos, la construcción de democracia participativa, etc.- y se planifican en abierto desafío al criterio de maximización de beneficios que domina en los medios privados-comerciales (Villamayor y Lamas, 1998: 216). Por otra parte, persiguen una función de servicio a la comunidad que es a veces desatendida por unos medios públicos, que, al menos en el sur de Europa, demuestran relaciones clientelares con respecto a gobiernos y grupos de presión (Hallin y Mancini, 2008), cuando no externalizan sus programaciones en la línea del «alarmante proceso de desmantelamiento del estado de bienestar» que acontece en España y otras partes del continente (Fernández Alonso, 2013: 28-29).

Los primeros medios comunitarios emergen en España en el contexto de la Transición, la cual favoreció un nuevo marco de oportunidades para la generación de alternativas comunicacionales críticas y desligadas de organismos públicos y corporaciones privadas. En distintos puntos de la geografía española surgieron proyectos ligados a nuevos actores como asociaciones vecina- 
les, colectivos juveniles o movimientos sociales de distinto cuño: feminismo, ecologismo, insumisión, etc. Muchas de estas iniciativas pioneras estuvieron inspiradas por el movimiento de radios libres y piratas de los años 70 de países como Italia o Francia, que reclamaban una liberación del espacio radioeléctrico para emitir programación a favor de colectivos infrarrepresentados en la agenda de los medios generalistas (Pérez Martínez, 2009 y 2012).

Las décadas de los 80 y los 90 supusieron una época convulsa para estas iniciativas, marcada por la aparición y la desaparición constante de muchos medios en un panorama de alegalidad y de ausencia de políticas públicas de apoyo al sector. Los distintos repartos de frecuencias de gobiernos socialistas y populares sirvieron para consolidar las emisoras comerciales y, en menor medida, a cargo de organismos autonómicos y ayuntamientos (radios y televisiones locales y autonómicas), pero en ningún momento se reservó espacio radioeléctrico ni para las radios gestionadas por organizaciones sociales ni para otro tipo de emisoras -escolares, universitarias, culturales y educativas de tipo diverso, etc.-, que venían funcionado al margen de las regulaciones (García García, 2013: 112).

En los últimos años, distintos organismos internacionales (Parlamento Europeo, 2008; Consejo de Europa, 2008; UNESCO, 2008; etc.) vienen reclamando a los estados la necesidad de proteger e impulsar un sector ciudadano de medios que, a todas luces, se expande en paralelo a la creciente monopolización del sistema de medios comerciales. Por otra parte, y correlato de su creciente expansión a nivel internacional, algunos países han aprobado marcos regulatorios favorables para el ámbito, en ocasiones prestando apoyo público explícito para proteger y consolidar el tercer sector. ${ }^{1}$ En el caso español, los principales avances tienen que ver con la conformación de alianzas comunicacionales, como la creación a nivel estatal en 2005 de la Red de Medios Comunitarios (RemC), constituida legalmente como Federación de Asociaciones en 2009 y que cuenta en la actualidad con más de 40 proyectos asociados entre radios, televisiones y medios impresos y digitales. A lo largo de su primera década de vida, la ReMC ha contribuido a la consolidación del sector y, en especial, a las tareas de lobby político para la reclamación de regulaciones favorables. Estos esfuerzos han facilitado que, por vez primera en la historia democrática, el Estado y algunas

1. En América Latina, son destacables los casos de Venezuela con la ley de telecomunicaciones (2000); Uruguay, con la ley de radiodifusión comunitaria (2007); o Argentina, con la ley de servicios de comunicación audiovisual (2009). En Europa, son pioneras las regulaciones de Francia, con la loi sur la communication audiovisuelle de 1982, o Reino Unido, con la aprobación de la Broadcasting Act en 1990, modificada por la Community Radio Order de 2004. 
comunidades autónomas (por ejemplo Andalucía) se planteen políticas públicas favorables al tercer sector de los medios. De hecho, en 2010, la Ley General 7/2010 de la Comunicación Audiovisual recogió, por primera vez, la denominación de «medios comunitarios» $\mathrm{y}$ «sin ánimo de lucro», no sin importantes restricciones y sin que hasta la fecha actual se hayan desarrollado los reglamentos que exige el citado marco (Meda, 2012 y 2014; García García, 2013).

\section{La investigación sobre el tercer sector de la comunicación en España}

Los medios comunitarios y alternativos no han supuesto una línea regular de investigación por parte de la comunidad académica de la comunicación en España. Los primeros años de la Transición estuvieron marcados por la aparición de los primeros estudios (Bassets, 1981; Prado, 1983; Vidal Beneyto, 1979), continuados durante la década de los 90 por algunas investigaciones aisladas (Chaparro, 1998 y 2002). Hay que esperar hasta mediados de la década de 2000 para encontrar un fortalecimiento de los estudios sobre el sector, en especial de corte conceptual y de descripción de experiencias (Barranquero y Meda, 2015; Peñafiel, 2014; Mayugo, 2006), histórico (Pérez Martínez, 2009, 2012 a, 2012 b; Fleischman, Reguero y Sáez, 2009) y regulatorio (Bergés, 2012; Meda, 2012 y 2015; García García, 2015).

En la actualidad, distintos trabajos avalan que la comunicación para el cambio social está dejando de ocupar un lugar marginal y periférico en la agenda académica (Fernández Viso, 2012; Marí Sáez, 2013). Esto tiene que ver, por un lado, con las posibilidades que hoy brindan tecnologías de bajo coste (Internet, Web 2.0, dispositivos móviles, etc.) para la comunicación participativa en la red, y, en segundo lugar, con la «maduración comunicacional» de muchos movimientos sociales, asociaciones y $\mathrm{ONG}$, que se apropian de manera innovadora de las tecnologías a su alcance, en especial desde el ciclo renovado de protestas que emerge a partir del 15M (Barranquero, 2014) y su sólido movimiento ciberactivista (Tascón y Quintana, 2012).

Lo que se presenta a continuación son algunos resultados preliminares de la primera investigación financiada hasta la fecha sobre los medios del tercer sector a escala estatal, dentro de los cuales abarcamos iniciativas sin ánimo de lucro, abiertas a la participación de la ciudadanía y que se orientan a la promoción de los derechos humanos y el cambio social. Al amparo de una ayuda a la investigación del Centro Reina Sofía sobre Adolescencia y Juventud, los datos que se presentan forman parte del proyecto «Los jóvenes y el tercer sector de la comunicación en España», que reúne a un total de 14 investigadores de distin- 
tas comunidades autónomas en un período comprendido entre octubre de 2014 y octubre de 2015. Otros resultados finales serán difundidos en la web creada a tal efecto: http://jovenesytercersector.com.

\section{Objetivos del estudio: una cartografía pionera de los medios del tercer sector}

La investigación se estructura a partir de un objetivo principal: realizar una cartografía pionera en el sector que recopile y sistematice los medios actualmente existentes en el Estado español, partiendo de la premisa de que, hasta la fecha, no se contaba con un censo actualizado, más allá de algunos intentos parciales. Tal era el caso de un trabajo de Javier García García (2013), quien en el caso de la radiodifusión señalaba la existencia de entre 200 y 300 radios que podrían englobarse dentro del ámbito de las emisoras libres, comunitarias, educativas y universitarias, distribuidas entre: 1) al menos un centenar de radios libres y comunitarias; 2) una veintena de radios universitarias, con importante desarrollo desde la creación de la Asociación de Radios Universitarias (ARU); 3) un número oscilante de pequeñas emisoras escolares; 4) otras emisoras de difícil calificación (de carácter social o cultural, en centros penitenciarios, etc.).

Este objetivo principal se subdivide, a su vez, en tres secundarios. El primero busca conocer cuál es la distribución geográfica y los idiomas predominantes en los medios comunitarios en España, para vislumbrar qué comunidades autónomas tienen una mayor presencia de este tipo de iniciativas y de qué manera contribuyen estos medios a la visibilización de lenguas y dialectos considerados, en muchos casos, periféricos. El segundo objetivo implica la descripción del tipo de medio y las tecnologías que utilizan para su emisión con el fin de vislumbrar algunas prácticas tecnológicas predominantes en el sector. En tercer lugar, se intenta examinar cómo se autoperciben estos medios, para lo cual nos aproximamos a las denominaciones más frecuentes en el sector y su visión de los principales valores y misiones que acometen en relación con su entorno más cercano.

\section{Muestra y metodología}

En primer lugar se lleva a cabo un mapeo centrado tanto en el ámbito de los medios tradicionales (prensa, radio y televisión) como en las nuevas expresiones digitales: cibermedios, radio online, blogs informativos, etc. Cabe tener en 
cuenta que, al hablar de tercer sector de la comunicación, el universo seleccionado comprende tanto los medios comunitarios clásicos -generalmente radios y televisiones, de acuerdo a la definición normativa descrita en el primer epígrafe (REMC, 2009; AMARC, 2009)-, como a medios educativos y culturales (de escuelas, institutos y universidades) y medios con gestión en forma de cooperativa o economía social. En estos últimos casos, solo forman parte de la muestra aquellas iniciativas que están abiertas a la participación de la ciudadanía o de la comunidad educativa (profesores, alumnos, etc.), tanto en la producción de contenidos como en la toma diaria de decisiones. Para los proyectos online, se contemplan exclusivamente medios con cierta consolidación y una trayectoria de, al menos, un año.

El mapeo recopiló un total de 345 medios repartidos por toda la geografía española, a partir de una base de datos que incluyó información sobre nombre del medio, municipio, comunidad autónoma, web y datos de contacto. Esta base fue elaborada recabando datos de las páginas web de las diferentes redes de medios comunitarios, así como a través del contacto directo con los responsables de algunas iniciativas.

A partir del mapeo, se diseñó un protocolo de análisis que se aplicó en forma de encuesta autoadministrada y en línea, teniendo en cuenta algunos parámetros metodológicos que garantizan su calidad, fiabilidad y validez (Cea D’Ancona, 2005; Wimmer y Dominick, 2011). El cuestionario diseñado para tal fin comprende un total de 40 categorías y se divide en tres partes. La primera, Datos de identificación, tiene como objetivos catalogar mediante datos sociogeográficos los medios estudiados y establecer un mapa interactivo de medios del tercer sector que se trasladará a partir de 2016 a un espacio web en el que se proporcionarán datos sobre los medios existentes en cada territorio (contacto, localización geográfica idioma predominante, URL, tipo de medio, etc.), y que, una vez articulado, se compartirá con periodistas del tercer sector y la ciudadanía a fin de permitir su reciclaje y permanente actualización.

La segunda parte del cuestionario, o Datos de organización, contenidos y participación en el medio, intenta recoger características relevantes a la hora de evaluar la fisonomía del medio y el tipo de relación que este sostiene con respecto a la ciudadanía, las organizaciones e instituciones y la participación de la comunidad más cercana. Es por ello que se incluyen categorías como: misiones y valores; modelo formal de toma de decisiones; regulación que guía las prácticas del medio; tipo de financiación predominante; organizaciones, colectivos y grupos o poblaciones que disponen de programas propios, actúan como fuente, o participan en la producción de contenidos del medio; target geográfico y poblacional al que van dirigidos; herramientas empleadas para obtener 
feedback de la comunidad; nivel de producción (propio o ajeno) de contenidos y programación.

La tercera y última parte del cuestionario, denominada Jóvenes y tercer sector, intenta extraer datos significativos acerca de la relación entre estos colectivos y los medios alternativos, de acuerdo a categorías como: número aproximado de voluntarios y asalariados por tramos de edad; requisitos para que un joven forme parte del medio; tipo de acuerdos firmados con entidades públicas, privadas o comunitarias para la formación o inserción profesional o social de los jóvenes; formación reglada y no reglada predominante de los jóvenes que participan en el medio; procesos de enseñanza/aprendizaje que ofrece el medio; tipos de programación dirigidos o elaborados por jóvenes; tareas más frecuentes en las que se desempeñan estos colectivos.

La encuesta fue enviada a la totalidad del universo de 345 medios de los que se obtuvo un índice de retorno de 94 cuestionarios. De este modo, y con un intervalo de confianza del $95 \%$, se garantiza una fiabilidad del 91,3\%, con un error muestral del 8,7\% (asumiendo pq $=0,50$ ). El pilotaje de la encuesta se había realizado previamente en 30 de ellos, y con este se validó la eficiencia del instrumento o protocolo (Barranquero y Meda, 2013).

\section{Distribución geográfica, idiomas y territorios}

La cartografía de medios comunitarios ha generado un catálogo de 345 medios con presencia en todas las comunidades autónomas del Estado, a excepción de La Rioja, Ceuta y Melilla. En la tabla 1 puede observarse la distribución geográfica de las iniciativas, en la que se constata que las comunidades autónomas con mayor penetración de este tipo de medios son, por este orden: Catalunya (36 \%), la Comunidad de Madrid (13\%) y Andalucía y Euskadi (9\% ambas).

En la misma tabla se comparan estos datos con los de los medios que respondieron a la encuesta. Mediante este cotejo, se comprueba el grado en que cada uno de los territorios del Estado está representado en la presente investigación. Como puede observarse, Catalunya (10 \%), Aragón (3\%) y Castilla y León e Illes Balears (1\%) son las regiones más subrepresentadas, en tanto que la Comunitat Valenciana (9\%), la Comunidad de Madrid (4\%), Andalucía ( $2 \%)$ y Galicia figuran entre los territorios más sobrerrepresentados. Por otro lado, cabe tener en cuenta algunas pequeñas desviaciones a la hora de interpretar el resto de resultados, por lo que, en caso de continuar la investigación, convendría incidir en la recopilación de datos de comunidades autónomas con un 
menor grado de representación. Sin embargo, la suma de todas las desviaciones resulta en una desviación general nula, lo que indica que el grado de fiabilidad de la muestra es alta, a pesar de las desviaciones marginales.

Tabla 1. Distribución geográfica de los medios del tercer sector en el Estado español. El universo total de medios mapeados es de 345 y la muestra de 94.

En la última columna se muestran las desviaciones marginales y el total de la muestra con respecto al universo

\begin{tabular}{|c|c|c|c|c|c|}
\hline \multirow[b]{2}{*}{ Andalucía } & \multicolumn{2}{|c|}{$\begin{array}{l}\text { Universo } \\
\mathbf{n}^{0} \text { medios } \\
\% \text { medios }\end{array}$} & \multicolumn{2}{|c|}{$\begin{array}{l}\text { Muestra } \\
\mathbf{n}^{0} \text { medios } \\
\% \text { medios }\end{array}$} & \multirow{2}{*}{$\begin{array}{c}\begin{array}{c}\text { Desviación } \\
\%\end{array} \\
2 \%\end{array}$} \\
\hline & 32 & $9 \%$ & 11 & $12 \%$ & \\
\hline Aragón & 14 & $4 \%$ & 1 & $1 \%$ & $-3 \%$ \\
\hline Asturias & 15 & $4 \%$ & 4 & $4 \%$ & $0 \%$ \\
\hline Illes Balears & 6 & $2 \%$ & 1 & $1 \%$ & $-1 \%$ \\
\hline Castilla-La Mancha & 4 & $1 \%$ & 1 & $1 \%$ & $0 \%$ \\
\hline Castilla y León & 21 & $6 \%$ & 5 & $5 \%$ & $-1 \%$ \\
\hline Canarias & 6 & $2 \%$ & 2 & $2 \%$ & $0 \%$ \\
\hline Cantabria & 1 & $0 \%$ & 0 & $0 \%$ & $0 \%$ \\
\hline Catalunya & 125 & $36 \%$ & 25 & $27 \%$ & $-10 \%$ \\
\hline Extremadura & 4 & $1 \%$ & 0 & $0 \%$ & $-1 \%$ \\
\hline Euskadi & 32 & $9 \%$ & 9 & $10 \%$ & $0 \%$ \\
\hline Galicia & 9 & $3 \%$ & 3 & $3 \%$ & $1 \%$ \\
\hline Com. de Madrid & 44 & $13 \%$ & 16 & $17 \%$ & $4 \%$ \\
\hline Región de Murcia & 7 & $2 \%$ & 1 & $1 \%$ & $-1 \%$ \\
\hline Navarra & 4 & $1 \%$ & 1 & $1 \%$ & $0 \%$ \\
\hline Com. Valenciana & 21 & $6 \%$ & 14 & $15 \%$ & $9 \%$ \\
\hline TOTAL & 345 & $100 \%$ & 94 & $100 \%$ & $0 \%$ \\
\hline
\end{tabular}

Fuente: elaboración propia

Por otra parte, también resulta interesante observar qué idiomas son los más comunes en sus programaciones, si entendemos que los medios comunitarios, libres y ciudadanos han desempeñado históricamente una importante labor en relación con el refuerzo de las identidades, expresiones culturales y lenguas, 
en especial de tipo minoritario y/o alternativas a la/s hegemónica/s, Los medios del tercer sector son considerados desde su potencial transformador no solo en discursos normativizados sino también de los procesos y relaciones sociales (Atton, 2002), puesto que profundizan la capacidad de expresión de la ciudadanía en general y de grupos minoritarios, en especial otorgando voz a los «sin voz» en el espacio público y generando con ello empoderamiento ciudadano (Ferron, 2012). Así entendidos, los medios de comunicación comunitaria se reconocerían como necesarios espacios de participación para aquellos que por su condición étnica, lingüística, cultural o política son marginados muchas veces y denigrados por el conjunto de los medios privados-comerciales, siendo reconocidos como uno de los pilares garantes del pluralismo cultural (AMARC, 2012).

El gráfico 1 demuestra cómo tan solo un $36 \%$ de los medios utiliza el castellano como lengua vehicular mientras que el resto lo usan en combinación con otro idioma cooficial del Estado o, incluso, idiomas no oficiales y dialectos. De hecho, un $20 \%$ utiliza en exclusiva el catalán, un $7 \%$ el euskera (o el euskera y el francés) y un $1 \%$ el gallego. Además, aparecen como idiomas vehiculares el asturiano y el aragonés que, a pesar de no estar reconocidos como lenguas cooficiales en sus territorios (Asturias y Aragón, respectivamente), cuentan con una masa considerable de hablantes.

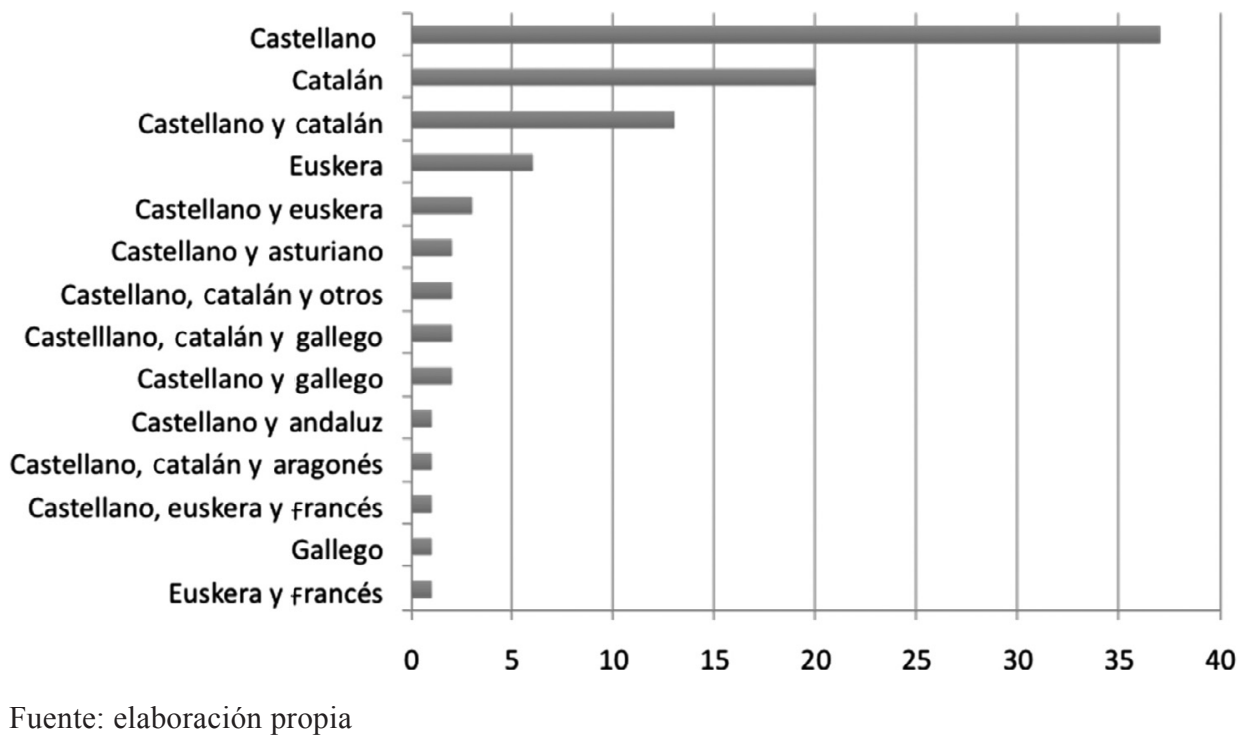

Gráfico 1. Idiomas vehiculares de los medios en el Estado español 


\section{Soportes y prácticas tecnológicas}

Otra de las dimensiones en las que incidió el estudio tiene que ver con los soportes en los que se vehiculan los mensajes de los medios comunitarios, entendiendo que tradicionalmente el medio radiofónico ha sido una de las plataformas más habituales de la comunicación para el cambio social, por potencialidades tales como su fácil acceso, su componente esencialmente oral y su fácil manejo en relación con soportes más complejos como los audiovisuales (como televisión). La tabla 2 muestra cómo el tipo de medio más frecuente es efectivamente la radio, con más de la mitad de medios. La siguiente plataforma es la prensa (tanto escrita como digital) y muy por detrás se sitúa la televisión, que solo es utilizada por el $11 \%$ de los medios comunitarios y alternativos. Con la rapidez de los avances tecnológicos acontecidos en los últimos años, destaca, por otro lado, la presencia de medios híbridos que podrían ser enmarcados en torno a un concepto más amplio de prácticas comunicacionales y que combinan distintos formatos e incluso modos de comunicación: mediática pero también grupal.

Tabla 2. Plataformas utilizadas por los medios comunitarios, libres y ciudadanos

\begin{tabular}{cc} 
Tipo de medio & \% del total \\
\hline Prensa & $34 \%$ \\
Radio & $55 \%$ \\
TV & $11 \%$ \\
\hline
\end{tabular}

Fuente: elaboración propia

Conocidas las plataformas y soportes que utilizan los medios del tercer sector, nos preguntamos por el tipo de tecnologías que utilizan para sus emisiones. Como puede observarse en el gráfico 2, en el caso de las radios, la mayor parte utiliza FM (un $44 \%$ sobre el total de medios, un $80 \%$ de las radios), aunque solo una minoría recurre a este canal como única vía de emisión. La mayoría de las radios complementan sus retransmisiones en FM con la producción de podcast y la emisión en streaming, complementando una tendencia internacionalizada sobre la transición en torno a la radiodifusión comunitaria a las tecnologías 
digitales (AMARC, 2010), entre las potencialidades y las brechas suscritas en la necesidad de construir procesos que fomenten la formación de las tecnologías para usos educativos y comunitarios, renovando los procesos de capacitación formales e informales y utilizando la fortaleza de las redes sociales y las tecnologías como formas de expansión y reivindicación propias; sin olvidarse, sin duda, del poder y la potencialidad de su combinación con lo analógico (AMARC, 2010). De hecho, la combinación de estos tres canales -FM, podcast y streaming- es la mayoritaria dentro de las radios (un $24 \%$ del total de los medios; un $44 \%$ de las radios).

En el caso de las televisiones, tan solo tres cadenas emiten en TDT, mientras que el resto lo hacen en streaming y utilizando vídeo en línea. Cabe aclarar que uno de los medios de la muestra, Radiotelevisió de Cardedeu, posee tanto radio como televisión, de manera que utiliza los cinco canales analizados. Sin embargo, a la hora de incluirlo en una plataforma, se decidió ubicarlo como televisión, debido a la preeminencia de este canal por encima de la radio.

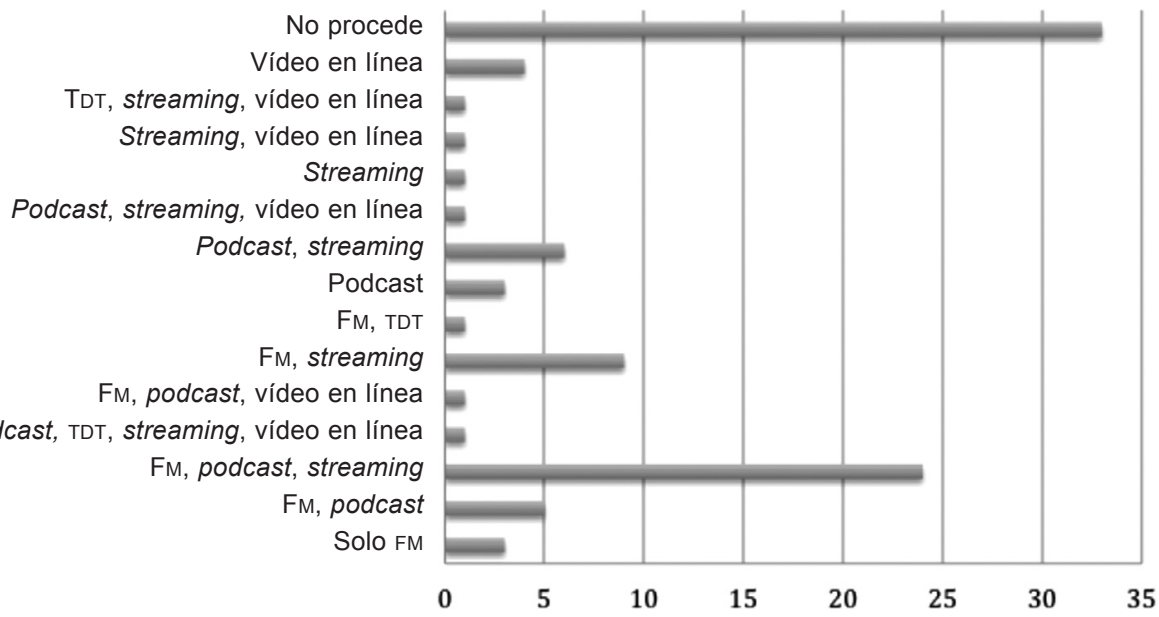

Fuente: Elaboración propia

Gráfico 2. Canales de emisión utilizados por los medios para el cambio en el Estado español. Se representa el \% de medios que utilizan cada una de las combinaciones posibles de canales. El $34 \%$ de medios etiquetados como «no procede» se corresponde a aquellos que son prensa escrita o digital 


\section{Identificación e autopercepción de los medios para el cambio}

Para conocer cómo se identifican y se autoperciben los medios comunitarios y alternativos se utilizaron tres variables diferentes: las redes a las que pertenecen -con el fin de conocer qué nexos establecen entre ellos y sus afinidades específicas-, cómo se autodefinen -con el objetivo de saber qué definiciones de las empleadas a nivel teórico, profesional y regulatorio se alinean con sus objetivos- y los valores y misiones que dirigen su trabajo.

En el gráfico 3 se muestran las redes de las que los medios son miembros o/y socios. Como puede observarse, un poco más de un $25 \%$ de los medios no está asociado a ninguna red, mientras que el resto lo está a una o más de ellas. La que acapara el mayor número de medios comunitarios y alternativos es la Red de Medios Comunitarios (REMC), a la que pertenece uno de cada cuatro medios, seguida de la red internacional Asociación Mundial de Radios Comunitarias (AMARC) a la que pertenecen el $11 \%$ de los medios. De estos datos se deduce que más de la mitad de los medios comunitarios del Estado español no están asociados a ninguna red y que prefieren relacionarse entre sí de acuerdo a agrupaciones (a veces informales) de alcance autonómico y regional, comarcal o incluso local. En algunos casos existen varias redes para un mismo territorio, como es el caso de la Comunidad de Madrid (Unión de Radios Libres y Comunitarias de Madrid, con años de trayectoria, y el Mercado Social Madrid) o Catalunya (Associació de Premsa Comarcal de Catalunya, Assemblea per la Comunicació Social y Ateneu Llibertari del Berguedà). Conviene destacar asimismo la presencia de la Asociación de Radios Universitarias (ARU), que congrega desde hace algún tiempo a las principales emisoras universitarias del Estado español, así como un conjunto de redes definidas de acuerdo a autonomías (Coordinadora de Radios Libres de Asturias, Rede Galega de Radios Libres e Comunitarias, etc.). Cabe desear, por otra parte, un mayor esfuerzo en el sentido de crear sinergias entre los medios. Esto podría constituir un acicate para el impulso y fortalecimiento del sector, en tanto que, a la luz de la encuesta, muchos medios continúan trabajando de forma aislada y con cierto desconocimiento con respecto al resto de iniciativas que componen el tercer sector de la comunicación.

No obstante, no hay que olvidar que, en muchas ocasiones, es necesario también comprender y tener en cuenta que, en momentos históricos de crisis y ruptura de la legitimidad institucional (De Certeau, 1974), la conformación de la movilización y la reivindicación de la protesta pasan en numerosas ocasiones por la construcción de redes informales que escapan a las estructuras históricas 
y se establecen a través de las redes personales, organizacionales o virtuales como mecanismos centrales en la consolidación del sector comunitario (Barranquero y Meda, 2015), con el caso reciente, para el territorio español, de las movilizaciones del $15 \mathrm{M}$ y el rol de los medios de comunicación comunitarios y alternativos, pero extrapolable también a otras experiencias dispersas por el resto del mundo (Mattoni y Treré, 2014; Gómez y Ramos, 2015; Gravante y Poma, 2013).

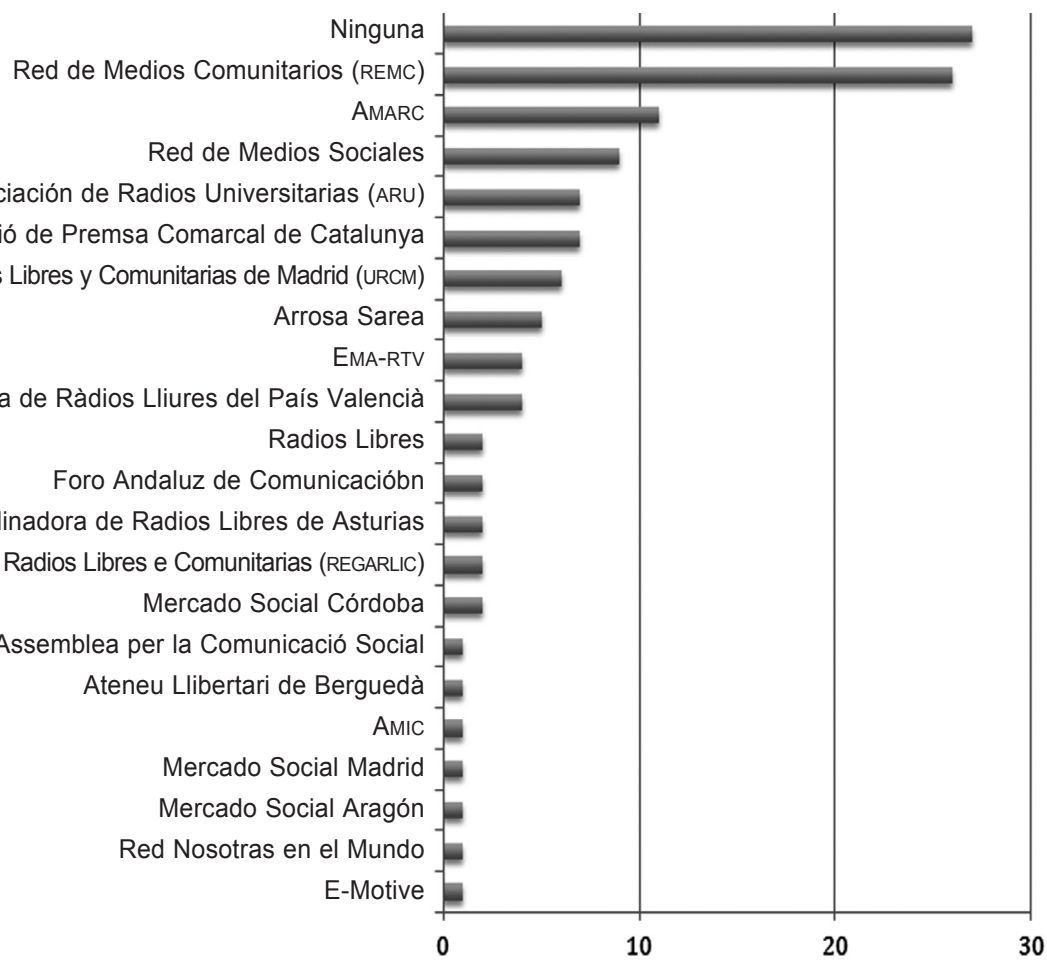

Fuente: Elaboración propia

Gráfico 3. Redes a las que se asocian y/o de las que son miembros los medios para el cambio en el Estado español. AMARC: Asociación Mundial de Radios Comunitarias; EMA-RTV: Asociación de Emisoras Municipales y Ciudadanas de Andalucía de Radio y Televisión; AMIC: Associació de Mitjans d'Informació i Comunicació 
Respecto a la autodefinición que los medios dan de sí mismos, es necesario remarcar que los proyectos encuestados eligieron su definición en torno a una pregunta multirrespuesta con un máximo de tres contestaciones sobre una lista cerrada de posibilidades. En el gráfico 4 se muestra cómo las mayoritarias se autodefinen como libres, de acuerdo a la tradición formalizada en torno al Manifiesto de Villaverde de 1983 -descrito en Pérez Martínez, 2012: 11- y comunitarias, definición propuesta, entre otros autores, por Rennie (2006) y Howley (2009) y mayoritaria en el ámbito de la regulación internacional (ej. AMARC), con las que se identifican más de un cuarto de los medios. Seguidamente aparece la definición de medios sin ánimo de lucro, propia de la normativa española, y locales, que se alinea con los debates habituales de la academia española en torno a los medios locales y de proximidad. Menos de un $15 \%$ se perciben como medios para el cambio social, definición que se fortalece a partir de la antología publicada por Gumucio Dagron y Tufte (2006), entre otros trabajos; independientes (Herman y Chomsky, 1988) o alternativos (Gumucio Dagron, 2004). Tan solo un $5 \%$ de los medios incluye entre sus definiciones el calificativo ciudadano (Rodríguez, 2001) o el complemento del tercer sector. Cabe destacar que ningún medio se definió como pirata.

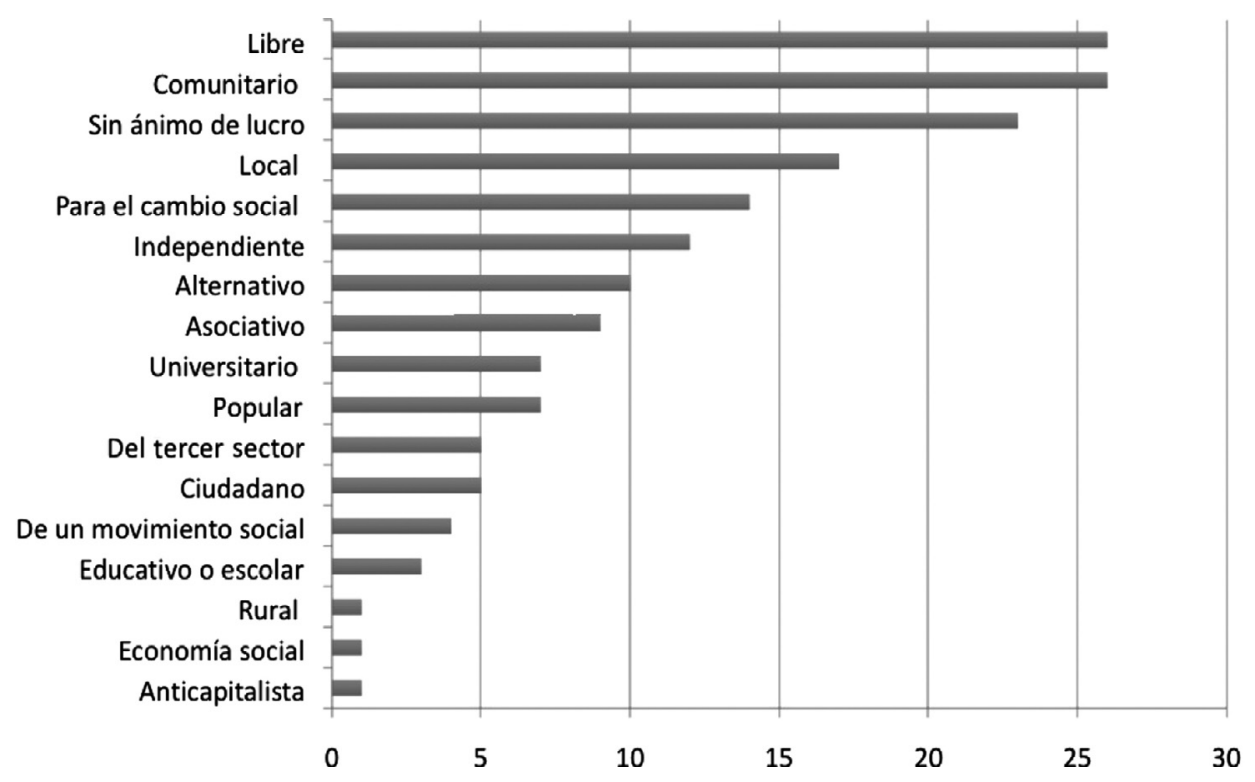

Fuente: elaboración propia

Gráfico 4. Autodefinición de los medios para el cambio en el Estado español 
Por último, en el Gráfico 5 se muestran las misiones y valores que defienden y rigen el trabajo de los medios comunitarios. Como puede observarse, más de la mitad de los medios se decantan por dar una información independiente como la más importante de sus misiones, seguida de ocio y tiempo libre y la animación cultural, que persiguen casi el $30 \%$ de los medios. En este sentido, conviene remarcar la paradoja de que, a pesar de que más de la mitad de los medios comunitarios del Estado no tienen el castellano como única lengua vehicular (ver el gráfico 1), solo un $3 \%$ de los medios tiene entre sus misiones la de favorecer el pluralismo y la diversidad cultural. En ese sentido, la explicación puede encontrarse si se toma en cuenta el carácter político y reivindicativo de las lenguas minoritarias y las culturas alternativas en un contexto de dominación cultural y epistemológica (De Certeau, 1974), siendo que, quizá, las contradicciones en los resultados se deriven en una multiplicidad mayor de respuestas, en consideración por parte de los medios comunitarios encuestados de la cultura como un elemento político y plural esencial, en la construcción social e identitaria de los colectivos y las comunidades en su sentido de «alternativa», incluso como herramienta de reivindicación.

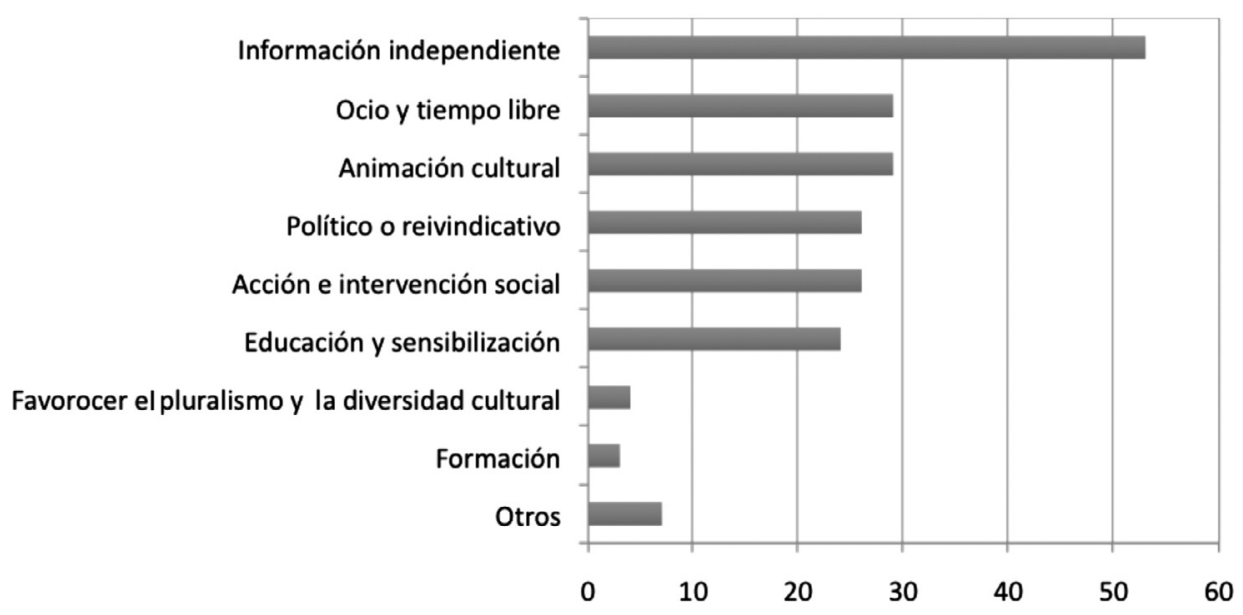

Fuente: elaboración propia

Gráfico 5. Misiones y valores de los medios para el cambio en el Estado español 


\section{Conclusiones}

Tras la exposición de los resultados, el presente trabajo arroja una serie de conclusiones que serán claves en la construcción de un elemento de investigación aglutinador y continuado en la comprensión de la organización, estructura y formas de participación y apropiación del sector de medios comunitarios en el Estado español.

En primer lugar, es necesario cerciorarse de que, ante la ausencia de estudios estructurales que dieran cuenta sobre la amplitud de actores presentes en el sector de los medios comunitarios, se ha descubierto que existe un universo amplio, heterogéneo y dinámico, constituido en torno a bloques de medios comunitarios históricos, pero también coyunturales, que ha poblado de manera más o menos desestructurada la geografía presente.

No obstante a la heterogeneidad suscrita en dicho universo de estudio, los sujetos analizados mostraron ciertas características confluyentes que ayudan a arrojar tendencias sobre la situación de dichos medios comunitarios, las cuales pasarían eminentemente por los siguientes puntos:

- En la construcción del sector y la distribución de los medios comunitarios. de manera estructural se establecen dos variables importantes a la hora de concentrar la existencia de dichos medios en unas regiones concretas. Tanto la densidad demográfica del territorio como la presencia de elementos distintivos culturales marcan de manera clara la distribución de los medios de comunicación en el Estado. Por tanto, podría concluirse que, dada la alta representación de medios en regiones como Catalunya, Euskadi o Galicia, la importancia de la existencia de lenguas y regiones culturalmente distintivas y propias ha sido un elemento relevante a la hora de conformar y encontrar experiencias de comunicación comunitaria. Dicha situación entronca con la necesidad suscrita de considerar estas lenguas y culturas alternativas a la dominante, tal y como muchos de los medios encuestados hace, como formas de acción y reivindicación política y síntoma del papel otorgado a los medios de comunicación comunitaria (AMARC, 2012) en la construcción del pluralismo cultural y mediático.

- En cuanto a las implicaciones de carácter tecnológico, a la certificación de los procesos de consolidación de las estructuras y medios digitales es necesario añadir la importancia presente y permanente de interesantes procesos de convivencia entre el digital y el analógico (AMARC, 2010), tomando en cuenta que la gran mayoría de los encuestados aún cuentan con ambas formas de transmisión y distribución en 
el aprovechamiento de las potencialidades diferenciadas presentes en cada una de las formas de interacción tecnológica.

- En cuanto a la consideración y construcción identitaria, es necesario rescatar la importancia del contexto y la relevancia del bloque histórico (Gramsci, 1980) como elemento aglutinador y de referencia epistémica, dado el autorreconocimiento con las estructuras y reivindicaciones por parte de la mayoría de los medios comunitarios encuestados de las consideraciones suscritas por el Manifiesto de Villaverde hace ya más de 30 años.

- A pesar de lo que pudiera pensarse, el estudio muestra una importancia relativa de las redes de medios, dado que, entre los encuestados, una importante mayoría dice no ser parte ni representar a ninguna de las redes constituidas formalmente en asociaciones de medios comunitarios. Más allá de una superficial consideración de la posibilidad de una desarticulación social de dichas estructuras, las formas de construcción y estructuración alternativas y propias suscritas históricamente por los medios de comunicación comunitarios, libres y ciudadanos hacen que, para futuras investigaciones sobre la situación del sector, sea quizá necesario ampliar los criterios metodológicos a fin de poder reconocer, de mejor manera y con mayor detalle, las formas de construcción informal de las interacciones presentes entre dichos medios.

\section{Referencias bibliográficas}

Amarc. Asociación Mundial de Radios Comunitarias (2009): Principios para un marco regulatorio democrático sobre radio y TV comunitaria, recuperado de: http://legislaciones.amarc.org/Principios/Principios_Legislacion_Radiodifusion_Comunitaria_esp.pdf.

- (2010): El bit de la cuestión. La radio popular y comunitaria en la era digital, Buenos Aires, AMARC-ALC/ALER.

- (2012): Derecho a la comunicación, pueblos indígenas y lenguas minoritarias, recuperado de: http://www2.amarc.org/?q=es/node/729.

Atton, C. (2002): Alternative media, London, Sage Publications.

Barranquero, A. (2014): «Comunicación, cambio social y ONG en España. Pistas para profundizar en la cultura de la cooperación desde los nuevos movimientos comunicacionales. El caso del 15M», Commons, revista comunicación y ciudadanía digital, 3 (1): 6-33.

Barranquero, A.; M. Meda (2015): «Los medios comunitarios y alternativos en el ciclo de protestas ciudadanas desde el 15M», Athenea Digital, 15 (1): 139-170, recuperado de: http://dx.doi.org/10.5565/rev/athenea.1385. 
BAssets, L. (ed.). (1981): De las ondas rojas a las radios libres, Barcelona, Gili.

Bergés, L. (2012): «Spain: An Information Society Without Traditional Offline Community Media?», Journal of Radio \& Audio Media, 19 (2): 134-151.

Cea D'Ancona, M. A. (2005): "La senda tortuosa de la "calidad" de la encuesta», Revista Española de Investigaciones Sociológicas (REIS), 111: 75-103, recuperado de: 10.2307/40184700.

Chaparro, M. (1998): Radio pública local, Madrid, Fragua.

- (2002): Sorprendiendo al futuro. Comunicación para el desarrollo e información audiovisual, Barcelona, Los Libros de la Frontera.

De Certeau, M. (1974): La cultura en plural, Buenos Aires, Nueva Visión.

Fernández Viso, A. (2012): «Historia de una travesía inconclusa: la comunicación para el desarrollo y el cambio social en la investigación y la docencia universitarias en España», CIC Cuadernos de Información y Comunicación, 17: 41-62, recuperado de: http://dx.doi.org/10.5209/rev_CIYC.2012. v17.39257.

Fernández Alonso, I. (2013): «Crisis financiera y medios públicos en España. Impacto de los recortes presupuestarios en las radiotelevisiones públicas autonómicas históricas (2008-2012)», Derecom, 12: 17-30, recuperado de: http://www.derecom.com/numeros/pdf/isabel.pdf.

Ferron B. (2012): «Giving voice to the voiceless or giving peace to the establishment? The ambivalent institutionalization of alternative minority media in Mexico and Israel» en Rigoni, I.; E. Saïtta (eds.): Minority Media in a Globalized Public Space, New York, Palgrave.

Fleischman, L.; N. Reguero; C. Sáez Baeza (2009): «Políticas de comunicación y sustentabilidad del tercer sector de la comunicación: el caso catalán en el contexto español y europeo», en Actas del VII Congreso Internacional de la Unión Latina de Economía Política de la Información, la Comunicación y la Cultura, ULEPICC: Políticas de cultura y comunicación: creatividad, diversidad y bienestar en la sociedad de la información, vol. 1, 1, Madrid, ULEPICC, 1-29.

García García, J. (2013): «Transformaciones en el tercer sector: el caso de las radios comunitarias en España», Adcomunica. Revista de estrategias, tendencias e innovación en comunicación, 5: 111-131, recuperado de: http://dx.doi.org/10.6035/2174-0992.2013.5.8.

Geerts, A.; V. VAn Oeyen (2001): La radio popular frente al nuevo siglo: estudio de vigencia e incidencia, Quito, ALER. 
Gómez, A.; J. Ramos (2015): «Medios Comunitarios en Bogotá. Indignación en tiempos de cólera», Revista Internacional de Comunicación y Desarrollo, 2: 149-168.

GramsCI, A. (1980): Notas sobre Maquiavelo, sobre la politica y sobre el Estado moderno, Buenos Aires, Nueva Visión.

Gravante, T.; A. Poma (2013): «Apropiación y emociones. Una propuesta para teórica desde abajo para analizar las prácticas del Net Activismo» en Sierra Caballero, F. (coord.): Ciudadanía, tecnología y Cultura. Nodos conceptuales para pensar la nueva mediación digital, Barcelona, Gedisa.

Gumucio Dagron, A. (2004): «The long and winding road of alternative media», en John Downing y otros (eds.): The Sage Handbook of Media Studies, London, UK \& Thousand Oaks, CA, Sage Publications, 41-63.

Gumucio Dagron, A.; T. Tufte (eds.) (2006): Communication for Social Change Anthology: Historical and Contemporary Readings, New Jersey, Communication for Social Change Consortium.

Hallin, D. C.; P. Mancini (2008): Sistemas mediáticos comparados. Tres modelos de relación entre los medios de comunicación y la política, Barcelona, Hacer.

Herman, E.; N. Chomsky (1988): Manufacturing Consent: The Political Economy of the Mass Media, New York, NJ, Pantheon.

Howley, K. (ed.) (2009): Understanding Community Media, Thousand Oaks, Sage.

Marí Sáez, V. (2013): «Comunicación, desarrollo y cambio social en España: entre la institucionalización y la implosión del campo», Commons, revista de comunicación y cultura digital, 2 (3): 40-63.

Mattoni, A.; E. Treré (2014): «Media practices, mediation processes and mediatization in the study of social movements», Communication Theory, 24 (3): 252-271.

MAYugo, C. (2006): «El "tercer sector audiovisual” irrumpe en la agenda política española», Pueblos. Revista de información y debate, 20, II Época.

Meda, M. (2012): «Del arte de cambiar para que todo siga igual: el tercer sector de la comunicación y la ley general audiovisual en España», Commons: revista comunicación y ciudadanía digital, 1 (1): 58-84.

- (2014): El tratamiento de los medios comunitarios en el marco de la ley general de la comunicación audiovisual, tesis doctoral no publicada, Universidad Complutense de Madrid.

Parlamento Europeo (2008): Resolución del Parlamento Europeo de 25 de septiembre de 2008, sobre los medios del tercer sector de la comunicación 
(TSC) (2008/2011(INI)), recuperado de:http:/www.europarl.europa.eu/sides/ getDoc.do?pubRef=-//EP//TEXT+TA+P6-TA-2008-0456+0+DOC+XML+v0//ES.

Pérez Martínez, J. E. (2009): «La radio libre española, aquel movimiento social olvidado», en Quirosa-Cheyrouze, R.; M. Fernández Amador (coords.): IV Congreso Internacional. Historia de la transición en España. Sociedad y movimientos sociales, Almería, Instituto de Estudios Almerienses, diputación de Almería, 897-914.

- (2012 a): «Libertad en las ondas. La radio libre madrileña» en Navajas, C.; D. Iturriaga (eds.): Coetánea: III Congreso Internacional de Historia de Nuestro Tiempo, Logroño, Universidad de La Rioja, 333-342.

- (2012 b): Una historia de la radio libre madrileña (1976-1989), documento de trabajo 2012/5 del Seminario de Investigación del Departamento de Historia Contemporánea, Universidad Complutense de Madrid, curso 2011-2012.

Peñafiel, C. (2014): «La radio libre y comunitaria en España: Un modelo social de comunicación alternativa» en Chaparro, M. (ed.): Medios de proximidad: participación social y políticas públicas, Girona y Málaga, Luces de Gálibo.

Prado, E. (1983): Las radios libres. Teoría y práctica de un movimiento alternativo, Barcelona, Mitre.

RENnie, E. (2006): Community media: a global introduction, New York, Rowman \& Littlefield.

Rodríguez, C. (2001): Fissures in the mediascape. An internacional study of citizens' media, Cresskill, NJ, Hampton.

Remc-Red de Medios Comunitarios (2009): Estatutos de la Red de Medios Comunitarios (RемC), recuperado de: http://medioscomunitarios.net/IMG/ pdf/ReMC_estatutos.pdf.

Tascón, M.; Y. Quintana (2012): Ciberactivismo: las nuevas revoluciones de las multitudes conectadas, Madrid, Catarata.

Vidal Beneyto, J. (ed.) (1979): Alternativas populares a las comunicaciones de masas, Madrid, Centro de Investigaciones Sociológicas-CIS.

Villamayor, C.; E. Lamas (1998): Gestión de la radio comunitaria y ciudadana, Quito, AMARC y FES.

Wimmer, R.; J. DominicK (2001): Introducción a la investigación en medios masivos de comunicación, Madrid, Ediciones Paraninfo. 